\title{
Antibiogram of Acinetobacter spp. Isolates in a Tertiary Care Hospital: A Lead towards Antibiotic Stewardship
}

\author{
B Apoorva*, Sneha Mohan, Tarana Sarwat and Dalip K Kakru \\ Department of Microbiology, SMS\&R, Sharda University, India \\ *Corresponding Author: B Apoorva, M.Sc. Medical Microbiology, Department \\ of Microbiology, School of Medical Sciences and Research, Sharda University, \\ Greater Noida, Uttar Pradesh, India.
}

Received: March 23, 2020

Published: April 17, 2020

(C) All rights are reserved by B Apoorva.

\begin{abstract}
Acinetobacter spp. is an important nosocomial pathogen especially in intensive care settings and is resistant to commonly available antimicrobial agents. Active surveillance is therefore necessary in order to determine appropriate antibiotic for the treatment. The purpose of this study was to determine the antibiogram of Acinetobacter spp. isolated in patients attending the tertiary care hospital. It is a prospective study conducted in the Department of Microbiology, Sharda Hospital.

Acinetobacter from various clinical samples were included in this study during six months period from May 2019 to 0 ctober 2019. The isolates were identified using conventional and automated methods (Vitek2 COMPACT, bioMérieux) and the susceptibility was done using the Kirby-Bauer disk diffusion assay. During the study period, a total of 40 Acinetobacter spp. were isolated from various clinical specimens, out of which $47.5 \%$ isolation was from ICUs. Highest isolation was observed from pus samples (22.5\%) followed by blood (17.5\%) and endotracheal aspirate (17.5\%). 77.5\% isolates were MDR (Multidrug Resistant), however they remained susceptible to colistin and tetracycline. It is necessary to regularly monitor the resistance phenotypes of Acinetobacter. Enhanced surveillance of MDR Acinetobacter is critical for guiding the rational use of antibiotics and reducing the incidence of hospital acquired infections.
\end{abstract}

Keywords: Acinetobacter spp; Multi-Drug Resistance (MDR); ICU; Antibiotic Susceptibility

\section{Abbreviation}

ICU: Intensive Care Unit; MDR: Multi-Drug Resistant; OPD: Out Patient Department; ICCU: Intensive Coronary Care Unit; HIC: Hospital Infection Control; BAL: Bronchioalveolar Lavage; CVP: Central Venous Pressure; NICU: Neuro Intensive Care Unit; RICU: Respiratory Intensive Care Unit; MICU: Medicine Intensive Care Unit

\section{Introduction}

Acinetobacter spp. are Gram negative, strictly aerobic, nonfastidious, non-fermentative coccobacilli, occurring in diploid formation, or in chains of variable lengths. They are non-motile, oxidase negative and catalase positive [1]. Acinetobacter spp. are recognised as an important nosocomial pathogen, particularly in patients admitted to Intensive Care settings (ICUs) and in immunocompromised patients [2]. These remain as one of the most challenging pathogens owing to their uniqueness and multiplicity of their resistance mechanisms [3]. Some of the risk factors for acquisition of infection by Acinetobacter spp. include prolonged hospitalization, immunocompromised status of patients, mechanical ventilation, cardiovascular or respiratory failure, previous infec- tion and antimicrobial therapy, and presence of indwelling catheters such as central venous or urinary catheters [1].

Acinetobacter spp. as pathogens are developing resistance at a very rapid pace to almost all antimicrobial agents that are available which includes, aminoglycosides, quinolones and broad-spectrum $\beta$ lactams [4]. Almost, $60-70 \%$ of these bacteria have developed resistance to many antibiotics, including carbapenems. And they are associated with higher patient morbidity, attributable mortality, and few or no antimicrobials remain effective for their treatment [5].

\section{Materials and Methods}

It is a prospective study carried out in the Sharda Hospital and Department of Microbiology, School of Medical Sciences and Research, Sharda University, Greater Noida. It was conducted for a period of 6 months from $1^{\text {st }}$ May 2019 - $31^{\text {st }}$ October 2019.

Forty (40) isolates of Acinetobacter spp. were recovered from various clinical specimens, namely pus (09 samples), blood (07 samples), Endotracheal Aspirate (07 samples), urine (06 samples), 
sputum (05 samples), Bronchioalveolar Lavage (BAL) (02 samples), Gluteal abscess (01 sample), throat swab (01 samples), CVP Tip (02 samples).

The isolates were speciated and their antimicrobial resistance pattern was studied. The samples received in the laboratory were inoculated on 5\% Sheep Blood Agar and MacConkey Agar and incubated overnight aerobically at both $37^{\circ} \mathrm{C}$ (to isolate Acinetobacter spp.). Thereafter species identification and in-vitro antibiotic susceptibility tests were performed. In case of urine samples, the isolates were subjected to biochemical tests and antimicrobial susceptibility only if the colony count was significant $\left(>10^{5} \mathrm{CFU} /\right.$ $\mathrm{ml}$ ). Acinetobacter spp. were identified by colony characteristics (Non-Lactose-fermenting, glistening, small mucoid colonies), Gram staining pattern and standard biochemical reactions (Catalase, Oxidase, Indole production, Citrate utilization, Motility, Urease activity, Reaction in Triple Sugar Iron medium).

After identification by phenotypic methods, antibiotic susceptibility was performed for each isolate by the Kirby-Bauer disc diffusion method on Mueller-Hinton Agar using 0.5 MacFarland Turbidity standard. The following antibiotic discs were used: Ampicillin (100 mcg), Piperacillin-tazobactam (100/10 mcg), ceftazidime (30 mcg), cefepime (30 mcg), ceftriaxone (30 mcg), cefotaxime (30 $\mathrm{mcg}$ ), doripenem (10 mcg), imipenem (10 mcg), meropenem (10 $\mathrm{mcg}$ ), gentamicin $(10 \mathrm{mcg})$, tobramycin $(10 \mathrm{mcg})$, amikacin (30 $\mathrm{mcg}$ ), ciprofloxacin (5 mcg), levofloxacin (5 mcg), tetracycline (30 $\mathrm{mcg}$ ), trimethoprim sulfamethoxole (25 mcg), colistin (0.016 - 256 $\mu \mathrm{g} / \mathrm{mL})$.

\section{Result}

A total of forty non-duplicated Acinetobacter strains (belonging to various species) were isolated from patients admitted and attending the OPD at Sharda Hospital, during the study period ( $1^{\text {st }}$ May 2019 to $31^{\text {st }}$ October 2019).

\section{Demographic profile}

Most of the patients from whom Acinetobacter spp. were isolated were in the age group of 0 - 9 years (22.5\%), followed by age group 20 - 29 years (17.5\%); 50 - 59 years (15\%); 40 - 49 years (12.5\%); 60 - 69 years $(12.5 \%) ; 30$ - 39 years $(10 \%) ; 70-79$ years $(7.5 \%) ; \geq 90$ years $(2.5 \%)$ respectively (Table 1$)$.

The patient's samples included in the present study had 20 Males (50\%) and 20 Females (50\%) (Figure 1).

Maximum number of Acinetobacter strains were recovered from pus 09 (22.5\%), followed by blood 07 (17.5\%); Endotracheal aspirate 07 (17.5\%); Urine 06 (15\%); sputum 05 (12.5\%); BAL, swab (Gluteal abscess and throat swab) and CVP tip 02 (5\%) each (Table 2).

\begin{tabular}{|c|c|c|}
\hline Age in Years & No. of isolates (N) & Percentage (\%) \\
\hline $0-9$ & 09 & 22.5 \\
\hline $10-19$ & 0 & 0 \\
\hline $20-29$ & 07 & 17.5 \\
\hline $30-39$ & 04 & 10 \\
\hline $40-49$ & 05 & 12.5 \\
\hline $50-59$ & 06 & 15 \\
\hline $60-69$ & 05 & 12.5 \\
\hline $70-79$ & 03 & 7.5 \\
\hline $80-89$ & 0 & 0 \\
\hline$\geq 90$ & 01 & 2.5 \\
\hline
\end{tabular}

Table 1: Age wise distribution of Acinetobacter.

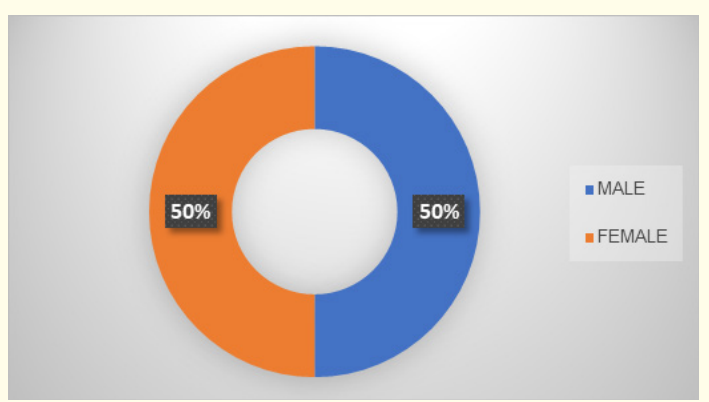

Figure 1: Gender wise distribution of Acinetobacter isolates.

\begin{tabular}{|l|c|c|}
\hline \multicolumn{1}{|c|}{ Sample } & No. of isolates (N) & Percentage (\%) \\
\hline Pus (Surgical wound) & $09(06)$ & $22.5(15)$ \\
\hline Blood & 07 & 17.5 \\
\hline Endotracheal Aspirate & 07 & 17.5 \\
\hline Urine & 06 & 15 \\
\hline Sputum & 05 & 12.5 \\
\hline BAL & 02 & 5 \\
\hline Swab (Gluteal abscess) & 01 & 2.5 \\
\hline Throat swab & 01 & 2.5 \\
\hline CVP Tip & 02 & 5 \\
\hline
\end{tabular}

Table 2: Sample wise distribution of Acinetobacter isolates.

Most of the isolates were obtained from patients admitted in the Intensive Coronary Care Unit (ICCU) 14 (35\%). Obstetrics and gynaecology $07(17.5 \%)$ were next in frequency followed by General Medicine 06 (15\%); General Surgery 03 (7.5\%) and paediatrics 03 (7.5\%). The least number of isolates were obtained from Neuro Intensive Care Unit 02 (5\%), Respiratory Intensive Care Unit 02 (5\%), Medicine Intensive care unit 01 (2.5\%), Burn unit 01 (2.5\%) and Orthopaedics 01 (2.5\%) (Table 3). 


\begin{tabular}{|l|c|c|}
\hline \multicolumn{1}{|c|}{ Ward } & No. of isolates (N) & Percentage (\%) \\
\hline ICCU & 14 & 35 \\
\hline Obs/Gyn & 07 & 17.5 \\
\hline General Medicine & 06 & 15 \\
\hline General Surgery & 03 & 7.5 \\
\hline Pediatrics & 03 & 7.5 \\
\hline NICU & 02 & 5 \\
\hline RICU & 02 & 5 \\
\hline MICU & 01 & 2.5 \\
\hline Burn Unit & 01 & 2.5 \\
\hline Orthopedics & 01 & 2.5 \\
\hline
\end{tabular}

Table 3: Ward wise distribution of Acinetobacter isolates.

Out of 40 isolates identified by Vitek 2, 82.5\% were Acinetobacter baumannii complex, followed by Acinetobacter Iwoffii and Acinetobacter haemolyticus 5\% each and Acinetobacter radioresistens, Acinetobacter ursingii and Acinetobacter junii 2.5\% respectively (Figure 2).

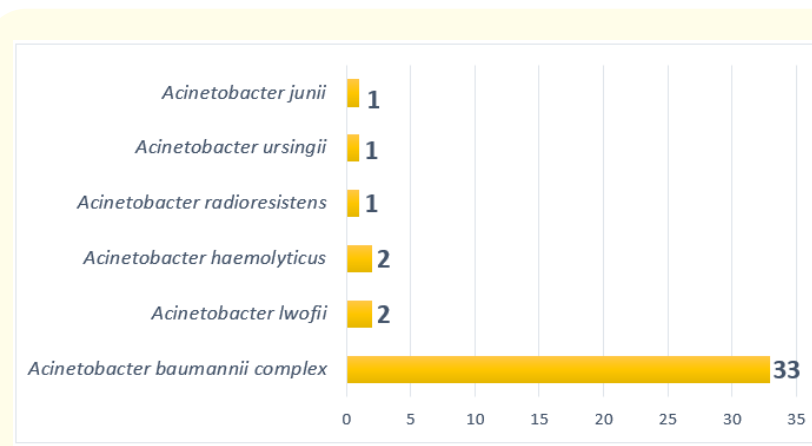

Figure 2: Species wise distribution of Acinetobacter isolates.

The Antimicrobial Susceptibility pattern of the various isolates are depicted in the table 4 and figure 3. 24/40 (60\%) and 22/40 $(55 \%)$ of isolates were resistant to carbapenems which is Meropenem and Imipenem respectively.

The isolates exhibited a high degree of resistance to piperacillin (92.5\%), $\beta$-lactam combination antibiotics such as PiperacillinTazobactam $29 / 40(72.5 \%)$ and cephalosporins. Out of 40 isolates, $32(80 \%)$ were resistant to cefotaxime and ceftazidime and cefepime and $31(77.5 \%)$ resistant to ceftriaxone.

There was a variable sensitivity to fluroquinolones. Thirty isolates $(75 \%)$, were resistant to ciprofloxacin, twenty-four isolates $(60 \%)$ to levofloxacin. In case of aminoglycosides, with amikacin resistance was seen in thirty-two $(80 \%)$ isolates, followed by tobramycin 26/40 (65\%) and in gentamicin it was seen in 22 (55\%) isolates.

\begin{tabular}{|c|c|c|}
\hline Antibiotic & Sensitive N (\%) & Resistant N (\%) \\
\hline Piperacillin & $03(7.5)$ & $37(92.5)$ \\
\hline $\begin{array}{c}\text { Piperacillin - } \\
\text { tazobactam }\end{array}$ & $11(27.5)$ & $29(72.5)$ \\
\hline Cefotaxime & $04(10)$ & $32(80)$ \\
\hline Ceftazidime & $05(12.5)$ & $32(80)$ \\
\hline Cefepime & $07(17.5)$ & $32(80)$ \\
\hline Ceftriaxone & $05(12.5)$ & $31(77.5)$ \\
\hline Imipenem & $11(27.5)$ & $22(55)$ \\
\hline Meropenem & $15(37.5)$ & $24(60)$ \\
\hline Ciprofloxacin & $08(20)$ & $30(75)$ \\
\hline Levofloxacin & $13(32.5)$ & $24(60)$ \\
\hline Gentamicin & $15(35.5)$ & $22(55)$ \\
\hline Tobramycin & $14(35)$ & $26(65)$ \\
\hline Amikacin & $08(20)$ & $32(80)$ \\
\hline Trimethoprim- & $09(22.5)$ & $27(67.5)$ \\
\hline sulfamethoxazole & & $0(0)$ \\
\hline Colistin & $40(100)$ & $0(0)$ \\
\hline Tetracycline & $06(100)$ & \\
\hline
\end{tabular}

Table 4: Antimicrobial susceptibility profile of Acinetobacter isolates.

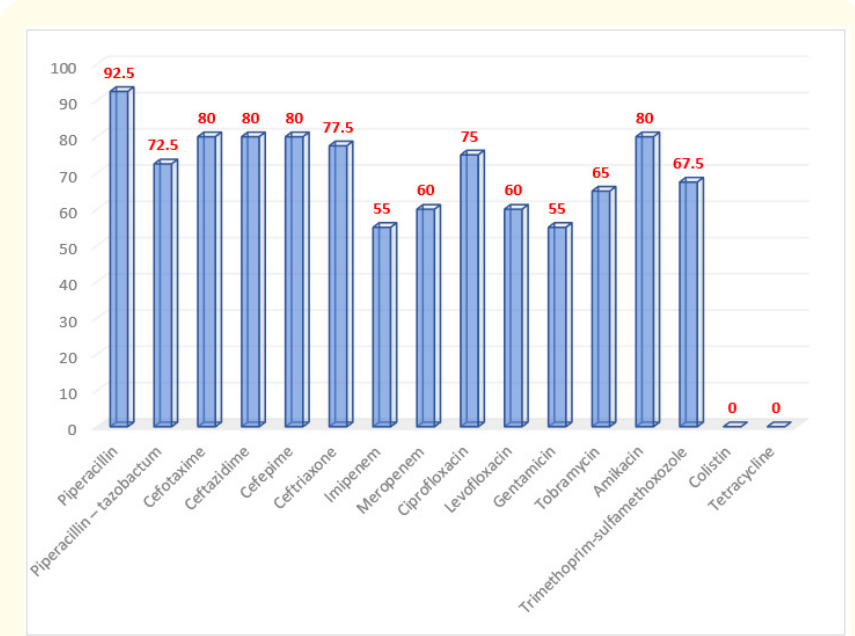

Figure 3: Antibiogram of Acinetobacter isolates (Resistant \%).

Tetracycline were tested against 6 isolates recovered from urine. None of the isolates $(0 / 6)$ were resistant against tetracycline. There were $100 \%$ sensitive.

All the isolates $40 / 40(100 \%)$ were sensitive to colistin. 
Out of 40 Acinetobacter isolates 31 (77.5\%) were MDR Acinetobacter, 02 (5\%) were resistant to 2 classes of antibiotics (penicillin and $3^{\text {rd }}$ generation cephalosporins) and $04(10 \%)$ were resistant to only 1 class of antibiotics (penicillin) (Table 5).

\begin{tabular}{|c|c|c|c|}
\hline $\begin{array}{c}\text { MDR } \\
\text { Acinetobacter } \\
\text { N (\%) }\end{array}$ & $\begin{array}{c}\text { Resistant to } \\
\text { piperacillin } \\
\text { only N (\%) }\end{array}$ & $\begin{array}{c}\text { Resistant to } \\
\text { piperacillin and 3 } \\
\text { generation } \\
\text { cephalosporins } \\
\text { N (\%) }\end{array}$ & $\begin{array}{c}\text { Sensitive } \\
\text { Acineto- } \\
\text { bacter } \\
\text { N (\%) }\end{array}$ \\
\hline $31(77.5)$ & $04(10)$ & $02(5)$ & $03(7.5)$ \\
\hline \multicolumn{3}{|c|}{ Total 40} \\
\hline
\end{tabular}

Table 5: Distribution of MDR Acinetobacter.

\section{Discussion}

Acinetobacter have emerged as one of the most significant pathogens causing nosocomial infections in patients admitted to Intensive Care settings [2]. Infections caused by multidrug resistant Acinetobacter are difficult to treat and are a major cause of increased morbidity and mortality in hospitalized patients [5]. The frequency of antibiotic resistance in Acinetobacter is worrisome since there are hardly any antibiotics in development process which have suitable activity against these multi-resistant strains of organism [6]. Until recently, carbapenem class of antibiotics were the drug of choice against this pathogen. However, with the development of resistance against carbapenems by Acinetobacter spp. the entire scenario has changed, making the pathogen difficult to treat [7].

The spread of such strains of Acinetobacter is a threat to the therapeutic options available and also a serious concern in the hospital infection control management. Such organisms are often difficult to treat and pose a significant risk due to their easy spread [7].

The present study was conducted to know the prevalence of Acinetobacter spp. infection in our hospital and to know their antibiotic susceptibility profiles and resistance patterns.

From amongst a total of 1,556 bacterial isolates cultured from various $(6,687)$ clinical specimens over a period of 6 months 40 $(2.5 \%)$ isolates were identified as Acinetobacter spp., similar prevalence of $3 \%$ and $3.36 \%$ of Acinetobacter isolates was reported by Dash., et al. in Odisha and Gupta., et al. in Pune [8,9]. Higher prevalence rate of $14 \%$ and $9.6 \%$ was reported by Mostofi., et al. in Tehran, Iran and Joshi., et al. in Pune [10,11].

Most of the patients in this study were in the age group 0 - 9 years $(22.5 \%)$ and $>60$ years $(22.5 \%)$ which was in concordance with the study conducted by Mera RM., et al. in United States, where the rise in isolation of Acinetobacter was seen among these age groups [12].

Maximum number of Acinetobacter strains in this study were isolated from pus $(09 / 22.5 \%)$, followed by blood $(07 / 17.5 \%)$; Endotracheal aspirate (07/17.5\%); urine (06/15\%); and sputum (05/12.5\%). A similar observation has been reported in the study done by Shivaranjani V., et al. in South India (2013) which showed $38.5 \%$ isolates from pus, followed by $20.4 \%$ isolates from endotracheal aspirate [13].

According to the literature, amongst Acinetobacter spp., commonest species isolated in human clinical specimens is Acinetobacter baumannii [14]. In this study also 33 (82.5\%) isolates were A. baumannii complex, followed by Acinetobacter lwoffii and Acinetobacter haemolyticus 02 (5\%) each and Acinetobacter radioresistens, Acinetobacter ursingii, Acinetobacter junii 01 (2.5\%) each. This again is in concordance with the study done by Gupta N., et al. in 2015 [9] where $72 \%$ were A. baumannii complex, followed by $A$. lwoffii, A. haemolyticus and $1 \%$ A. radioresistens, A. junii.

In the present study, Acinetobacter spp. were found to be resistant to most commonly used antimicrobial agents as a routine and prevalence of $77.5 \%$ MDR was observed. Similar reports of MDR Acinetobacter isolates have been reported with $88.02 \%$ resistance to commonly applied antibiotics [15]. Acinetobacter is ubiquitous in the hospital setting. It has the ability to survive for longer periods and also demonstrates a number of antimicrobial resistance genes which has made Acinetobacter a successful hospital pathogen [8].

Acinetobacter were extremely resistant to piperacillin (92.5\%) which correlates with the study done by Shivaranjani V., et al. in South India (2013) [13]. 32 (80\%) of isolates were also resistant to cephalosporins correlating with studies done by Guckan R., et al. in 2015 and Shivaranjani V., et al. in 2013 [13,16]. Resistance towards imipenem and meropenem was seen to be $55 \%$ and $60 \%$ respectively. Data of the antibiotic susceptibility patterns of Acinetobacter from different geographical areas revealed that the resistance of Acinetobacter spp. to imipenem rose from no resistance to $40 \%$ (2000 - 2004) [17]. The prevalence of imipenem resistance in A. baumannii isolated from a burns unit of the USA was $8 \%$ earlier (2007) [18]. Resistances to major antimicrobial drugs as well as disinfectants are the major factors that make it a successful and persistent hospital pathogen [19]. No resistance to colistin was seen in this study which is similar to the studies published by Dash., et al, Shareek., et al. and Nazir A $[8,19,20]$.

Initial concern about multidrug resistant (MDR) and carbapenem resistant Acinetobacter baumannii (CRAB) associated infections began when the first hospital wide outbreak occurred in New York 
City in 1991 [21]. Since then, reports of CRAB from other parts of the world including India [20,22] are coming in. Out of the total isolates $26(65 \%)$ were multidrug resistant (MDR) in this study. Other studies conducted by Dash., et al. (2013) and Rekha., et al. (2011) reported MDR isolates to be 55\% and 74\% respectively $[8,23]$.

Most of our Acinetobacter spp. were isolated from patients admitted in the high-risk settings like Intensive Coronary Care Unit (ICCU) 35\%. The results are concordant with the results seen in the study conducted by Mera RM., et al. (2010) and Gupta., et al. (2015), where an increased number of Acinetobacter isolates were recovered from Intensive Care settings [9,12]. The emergence of antibiotic resistant strains in ICU is because of higher use of antimicrobial agents per patient and per surface area [9].

\section{Conclusion}

Resistance rate of Acinetobacter to routinely used antibiotics is increasing rapidly. $57.5 \%$ and $67.5 \%$ isolates in this study were resistant to carbapenems and fluroquinolones respectively. MDR Acinetobacter isolates remained susceptible to colistin and tetracycline, which can be used as the treatment option for management of most of the cases of infections caused by this organism, however with caution as colistin is and should be a last resort. Patients infected with MDR Acinetobacter is widely spread in our hospital and occurred mostly in children below 10 years of age and in patients admitted to intensive care settings and the reason/s behind this alarming situation need to be ascertained and taken care of at regular intervals.

It is necessary to regularly monitor the resistance phenotypes of these isolates. Enhanced surveillance of MDR Acinetobacter is critical for guiding the rational use of antibiotics and reducing the incidence of Hospital Infection Control (HIC).

\section{Conflict of Interest}

There are no conflicts of interest.

\section{Bibliography}

1. Looveren MV Goossens H and the ARPAC Steering Group. "Antimicrobial resistance of Acinetobacter spp. in Europe". Clinical Microbiology and Infection 10 (2004): 684-704.

2. Arroyo LA., et al. "Reliability of the E-Test Method for detection of Colistin resistance in clinical isolates of Acinetobacter baumannii". Journal of Clinical Microbiology 43 (2005): 903905.

3. Wenzier E., et al. "Anticipating the Unpredictable: A Review of antimicrobial stewardship and Acinetobacter Infections". Infectious Diseases and Therapy 6.2 (2017): 149-172.
4. Tille PM. "Acinetobacter, Stenotrophomonas, and other Organisms". In Eds: Aziz HA, Klaassen JC, Heuertz RM., et al. Bailey and Scott"s Diagnostic Microbiology, 14 ${ }^{\text {th }}$ Edition. Elsevier, 3251 Riverpert Lane St. Louis, Missouri 63043 (2017): 361362.

5. Liu CP., et al. "Risk Factors for Mortality in Patients with Acinetobacter baumannii Bacteremia". Infection and Chemotherapy 45 (2013): 325-330.

6. Swathi CH., et al. "Direct determination and differentiation of carbapenemases of A. baumannii from uncultured tracheal samples". Bio Rxiv (2018).

7. Walsh TR., et al. "Metallo $\beta$-lactamases: the quiet before storm?" Clinical Microbiology Reviews 18 (2005): 306-325.

8. Dash M., et al. "Frequency, risk factors and antibiogram of Acinetobacter species isolated from various clinical samples in a tertiary care hospital in Odisha, India". Avicenna Journal of Medicine 3.4 (2013): 97-102.

9. Gupta N., et al. "Isolation and identification of Acinetobacter species with special reference to antibiotic resistance". Journal of Natural Science, Biology and Medicine 6 (2015): 159-162.

10. Mostofi S., et al. "Multi-drug resistance in Acinetobacter baumannii strains isolated from clinical specimens from three hospitals in Tehran-Iran". African Journal of Microbiology Research 5 (2011): 3579-3582.

11. Joshi SG., et al. "Clinical and demographic features of infection caused by Acinetobacter species". Indian Journal of Medical Sciences 60 (2006): 351-360.

12. Mera RM., et al. "Acinetobacter baumannii 2002-2008: Increase of Carbapenem Associated Multiclass Resistance in the United States". Microbial Drug Resistance 16 (2010): 209-215.

13. Sivaranjani V., et al. "Multi-drug resistant Acinetobacter species from various clinical samples in a tertiary care hospital from South India”. Australasian Medical Journal 6.12 (2013): 697-700.

14. Winn W., et al. "Non-fermenting Gram negative bacilli". In: Koneman'scolor Atlas and textbook of Diagnostic Microbiology. $6^{\text {th }}$ edition. USA: Lippincott Williams and Wilkins Company (2006): 305-391. 
15. Banerjee T., et al. "High Prevalence and Endemicity of Multidrug Resistant Acinetobacter spp. in Intensive Care Unit of a Tertiary Care Hospital, Varanasi, India”. Journal of Pathogens (2018): 9129083.

16. Guckan R., et al. "Antimicrobial susceptibility of Acinetobacter baumannii complex isolated from different clinical samples in a tertiary care hospital". Journal of Antibiotics Research 01.01 (2015).

17. Perez F., et al. "Global challenge of multidrug-resistant Acinetobacter baumannii". Antimicrobial Agents and Chemotherapy 51 (2007): 3471-3484.

18. Trottier V., et al. "Outcomes of Acinetobacter baumannii infection in critically ill burned patients". Journal of Burn Care and Research 28 (2007): 248-254.

19. Saleem AF., et al. "Pan-resistant Acinetobacter infection in neonates in Karachi, Pakistan". Journal of Infection in Developing Countries 4 (2009): 30-37.

20. Shareek PS., et al. "Antibiotic sensitivity pattern of blood isolates of Acinetobacter species in a tertiary care hospital: A retrospective analysis". American Journal of Infectious Diseases 8 (2012): 65-69.

21. Nazir A. "Miltidrug-resistant Acinetobacter septicaemia in neonates: A Study from a teaching hospital of Northern India". Journal of Laboratory Physicians 11 (2019): 23-28.

22. Urban C., et al. "Effect of sulbactum on infections caused by imipenem-resistant Acinetobacter calcoaceticus biotype anitratus". The Journal of Infectious Diseases 167 (1993): 448-451.

23. Rekha S., et al. "Multidrug resistant Acinetobacter isolates from patients admitted at Kolar". Journal of Clinical and Biomedical Sciences 1 (2011): 3-7.

\section{Assets from publication with us}

- Prompt Acknowledgement after receiving the article

- Thorough Double blinded peer review

- Rapid Publication

- Issue of Publication Certificate

- High visibility of your Published work

Website: https://www.actascientific.com/

Submit Article: https://www.actascientific.com/submission.php Email us: editor@actascientific.com

Contact us: +919182824667 